\title{
March 2016 Arizona Thoracic Society Notes
}

The March 2016 Arizona Thoracic Society meeting was held on Wednesday, March 23, 2016 at the Scottsdale Shea Hospital beginning at 6:30 PM. This was a dinner meeting with case presentations. There were 17 in attendance representing the pulmonary, critical care, sleep, and radiology communities. Of note, Dr. Elijah Poulos drove from Flagstaff to attend the meeting.

Dr. Rick Robbins gave a summary of ATS Hill Day and the possibility of collecting dues for the Arizona Thoracic Society along with American Thoracic Society dues. Dr. Robbins also presented the results of emailing the Table of Contents of the Southwest Journal of Pulmonary and Critical Care to the ATS members in Arizona, New Mexico, Colorado, and Nevada along with listing the contents in Inspirations the California Thoracic Society newsletter. The number of page views doubled over usual the following day.

Dr. George Parides presented a short presentation on whether coccidioidomycosis nodules in the setting of biologics for rheumatoid arthritis should receive fluconazole and the new coccidioidomycosis skin test under development.

Dr. Lewis Wesselius presented a plaque to Dr. Robbins who was voted 2016 Arizona Thoracic Society clinician of the year (Figure 1).

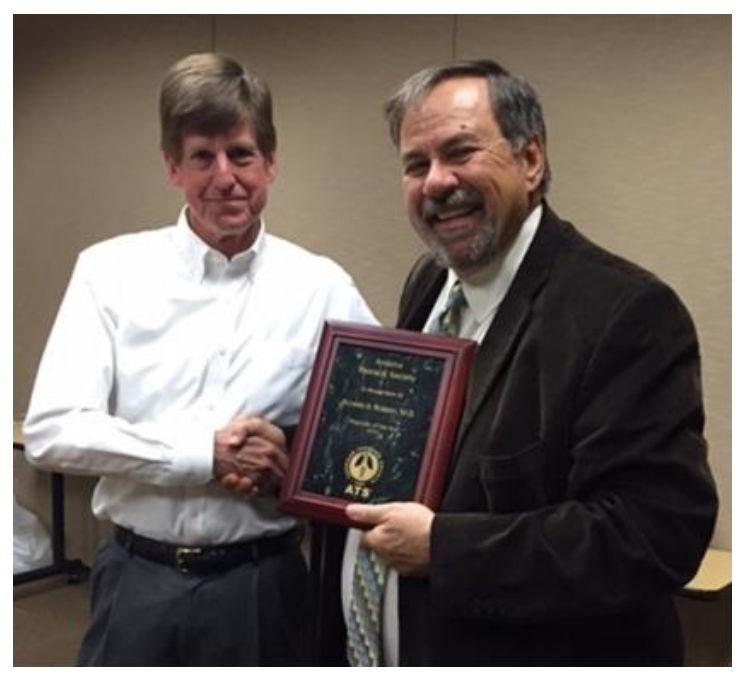

Figure 1. Dr. Lewis Wesselius (left) presenting a plaque to Dr. Rick Robbins.

There were 5 case presentations:

1. Dr. George Parides presented a 67-year-old man with a thin walled cavity and positive coccidioidomycosis serology who was unable to tolerate fluconazole and voriconazole. There were several possible therapies suggested including posaconazole or resection of the cavity. 
2. Dr. Elijah Poulos presented a case of 44-year-old woman who had occupational exposure to mineral spirits and presented with a chronic dry cough. Chest x-ray showed bilateral apical infiltrates. Thoracic CT scan confirmed the presence of the infiltrates which appeared lobular. Physical examination and laboratory evaluation including induced sputum specimens were unrevealing. A bronchoscopy with bronchoalveolar lavage and transbronchial biopsy were performed. The biopsy was consistent with acute eosinophilic pneumonia. Because her cough and CT scan were improving no therapy was given. A follow-up CT scan showed resolution of the apical consolidations but a new rounded $4 \mathrm{~cm}$ area of consolidation but her cough has resolved and she is now asymptomatic. The group suggested several possibilities including possible lipoid pneumonia or possible cryptogenic organizing pneumonia. The majority felt that following the patient was the most appropriate course of action.

3. Dr. Paul Conomos presented a case of a 43-year-old man who had an incidental finding of a vessel in the left lower lung originating from the abdominal aorta. The abdominal CT scan was performed for abdominal pain which quickly resolved. He had no respiratory symptoms. It was that this was likely a pulmonary sequestration and discussions with invasive radiology to better define the vascular supply and thoracic surgery for possible resection might be useful.

4. Dr. Lewis Wesselius presented a case of an 65-year-old woman who presented to her gastroenterologist with anemia and some weight loss. A thoracic CT scan was performed which suggested a tracheal abnormality, possibly a tracheal wall lesion. Bronchoscopy showed a smooth indentation in the trachea with a yellowish discoloration. A similar yellowish area was seen near the main carina. Biopsies were performed. Congo red stain was positive consistent with amyloidosis.

5. Dr. Allen Thomas presented a 62-year-old man with symptoms of an upper respiratory infection beginning in January who improved sufficiently that he rode his motorcycle near Bagdad, AZ this month. He rode through a dust storm and subsequently developed dyspnea, cough and gray sputum production. Laboratory evaluation in an emergency department showed a pO2 of 60 on room air but was otherwise unremarkable. He was seen in pulmonary consolidation a few days later. Thoracic CT scan showed subpleural areas of ground glass and consolidation. The patient was asymptomatic by this time and declined biopsy. The group suggested following the patient with a repeat thoracic CT scan. It was suggested that this could possibly be a case of acute eosinophilic pneumonia.

There being no further business, the meeting was adjourned about 8 PM. The next meeting will be in Phoenix on Wednesday, May 25,2016 at 6:30 PM.

Richard A. Robbins, MD Editor, SWJPCC 